\title{
Senza una multidisciplinarietà organizzata
}

\author{
Roberto Fanelli ${ }^{1,2}$, Marco Lombardi ${ }^{1,2}$, Stefano Michelassi' ${ }^{2}$, Carmela Zizzo ${ }^{3}$, Giovanni Duro ${ }^{3}$ \\ ${ }^{1}$ SOS Emodialisi del Mugello, Ospedale Nuovo del Mugello, Azienda USL Toscana Centro, Borgo San Lorenzo (FI) \\ ${ }^{2}$ SOC Nefrologia e Dialisi, Ospedale SM Annunziata, Azienda USL Toscana Centro, Firenze \\ ${ }^{3}$ Istituto di Biomedicina ed Immunologia Molecolare "A. Monroy", del CNR-Palermo, Palermo
}

\begin{abstract}
The importance of multidisciplinarity
Anderson-Fabry disease is a multisystem disease associated with major complications of the organs involved. This case report describes the turbulent course the disease may take in some patients and underscores the need for clinical and diagnostic protocols that encourage cooperation between different clinicians. The treating nephrologist in the present case was thwarted in his efforts to accomplish a collegial reassessment of the patient together with clinicians of the Anderson-Fabry referral center. Ideally, such a complex patient would have to be managed by a team of specialists interacting with each other in (almost) real time.
\end{abstract}

Keywords: Anderson-Fabry disease, Communication, Multidisciplinarity, Multisystem disease

\section{Il caso clinico}

Nel novembre 2014 fu trasferito nel nostro Centro dialisi un paziente affetto da malattia di Anderson-Fabry (AF).

Si trattava di un uomo di razza caucasica di 54 anni (nato nel 1960), con storia familiare di AF e cardiopatia ischemica. Ex fumatore, nel 1987 era stato sottoposto a colectomia totale con risparmio dell'ampolla rettale per poliposi multipla del colon ma per il resto riferiva apparente buona salute fino al 1990. Da quell'anno aveva sofferto per frequenti episodi bronchitici acuti a carattere asmatiforme e test allergologici erano risultati positivi ad antigeni multipli (polveri, ulivo, cipresso, parietaria, pelo del gatto). Nello stesso periodo di tempo erano sopravvenuti ricorrenti episodi ischemici cerebrali transitori. Nel 2000 era stata riscontrata ipertensione arteriosa e nel 2005 era stata riconosciuta una malattia renale cronica (MRC) (valori di creatininemia e di proteinuria non disponibili) che, indagata (senza aver eseguito una biopsia

\footnotetext{
Accepted: May 28, 2017
}

Published online: September 5, 2017

Indirizzo per la corrispondenza:

Dr. Marco Lombardi

Nefrologia e Dialisi

Ospedale del Mugello

Viale della Resistenza, 60

50032 Borgo San Lorenzo (FI)

lombardim@tin.it renale), aveva portato alla diagnosi di AF. Nello stesso anno il paziente aveva iniziato trattamento enzimatico sostitutivo (ERT) presso il Centro regionale di riferimento per AF, inizialmente con agalsidasi-beta e.v. (70 mg ogni 14 giorni) e da novembre 2009, per indisponibilità del farmaco, con agalsidasialfa e.v. (14 mg ogni 14 giorni). Per progressione della MRC il paziente era stato inserito in lista di trapianto renale, nel 2010 venivano confezionate una prima fistola artero-venosa (FAV) radio-cefalica distale per dialisi, complicata da trombosi precoce, e quindi una seconda FAV prossimale alla precedente. Nel 2011 era stato iniziato trattamento emodialitico cronico e nei tre anni successivi aveva cambiato tre Centri dialisi. Nel 2013 decise di uscire dalla lista trapianto. All'epoca il Centro regionale di riferimento aveva certificato le seguenti manifestazioni di AF nel paziente:

- Insufficienza renale cronica (IRC) in terapia sostitutiva mediante emodialisi

- Neuropatia delle piccole fibre

- Neuropatia delle grosse fibre per accumulo da AF e da IRC-dialisi

- Cardiopatia ipertrofica

- Angiocheratomi

- Cornea verticillata

Trasferito per motivi logistici nel nostro Centro nel novembre 2014, il decorso era complicato dalla comparsa di altre manifestazioni di $A F$, fra cui una sindrome delle gambe senza riposo, ipoacusia con acufeni e crisi dolorose intollerabili alle 


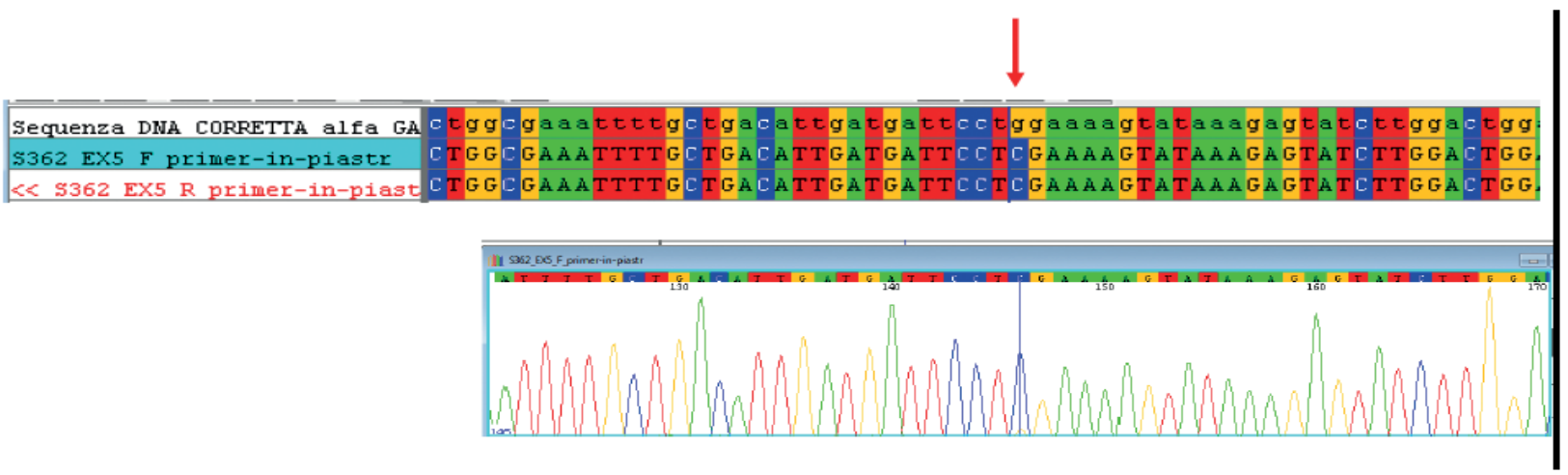

Fig. 1 - La figura mostra l'allineamento delle sequenze Forward e Reverse dell'esone 5 del campione in esame, rispetto alla sequenza wt del gene GLA (sequenza DNA CORRETTA alfa GAL A). II punto indicato dalla freccia si riferisce al sito in cui ricade la mutazione c.707 G>C che nella sequenza wt è una Guanina mentre nel campione in esame (filamento Forward e Reverse) è una Citosina. Questo dato è ulteriormente confermato dall'elettroferogramma (filamento Forward) in cui al posto del picco giallo (guanina) vi è un picco blu corrispondente a una citosina, indicato dalla linea blu.

estremità e successivamente anche al volto. Nel febbraio 2015, nell'ambito di accertamenti per rapida anemizzazione, il paziente era sottoposto a rimozione endoscopica di 5 polipi sessili uno dei quali maligno (adenocarcinoma intramucoso su sfondo di adenoma tubulo-villoso di alto grado) e nell'agosto dello stesso anno veniva effettuato intervento di proctectomia con anastomosi ileopauchanale e ileostomia di protezione, complicata da deiscenza dell'anastomosi e laparocele. La TC di controllo evidenziava una massa renale sinistra di possibile natura neoplastica e in dicembre veniva eseguito intervento di nefrectomia sinistra associato a intervento di contenzione di laparocele e revisione di fistola anastomotica con J-pouch ileo-anale. Nel frattempo il paziente aveva avuto anche gravi problemi di accesso vascolare. Nel 2014, per trombosi della FAV su vasi nativi, era stata confezionata una FAV protesica omero-omerale sinistra che due anni dopo, per trombosi e formazione di pseudoaneurisma, veniva rimossa e sostituita con catetere venoso centrale permanente (CVCp) in vena giugulare destra.

Nei due anni trascorsi presso il nostro Centro le condizioni generali del paziente peggioravano ulteriormente con comparsa di grave astenia e inefficacia delle terapie proposte per le crisi dolorose (carbamazepina, paracetamolo). In aprile 2016 una infiammazione (dolore, eritema, calore, edema) localizzata ai tessuti del carpo, associata ad accessi febbrili, elevati markers di flogosi (PCR, ferritina, fibrinogeno) e autoimmunità negativa (ANA, WR, RA test, C3, C4), era attribuita a panniculite e trattata (con scarsa efficacia) con antibiotici e steroidi. Pochi mesi dopo comparivano anche dolori al rachide cervicale e lesioni papulari chiare sulla fronte attribuite a deposizione microcristallina e parzialmente migliorate con colchicina (uricemia costantemente inferiore a $6 \mathrm{mg} / \mathrm{dL}$ ). II dosaggio enzimatico dell'alfa-galattosidasi-A risultava pressoché assente e nell'esone 5 del gene GLA veniva rivelata la mutazione $c .707 G>C$, sostituzione di una guanina con una citosina in posizione $707 \mathrm{del}$ c.DNA che determina la sostituzione di un triptofano con una serina in posizione 236 della proteina (Fig. 1) (1, 2), mai descritta precedentemente. La stessa ricerca era stata fatta con medesimo risultato nel 2005 dal Centro di riferimento ma non era stato possibile recuperarla dal paziente.

Venivano anche dimostrati un accumulo di Lyso-Gb3 $(46,4$ $\mathrm{nMol} / \mathrm{L}$ [VN 0,08-1,13 nMol/L]). La ricerca della malattia nel fratello risultava negativa (dosaggio enzimatico $4,7 \mathrm{nmoli} / \mathrm{h} / \mathrm{mL}$ [vn >3]). Non siamo riusciti a ottenere dati circa la madre (affetta) e la sorella di cui non è noto neppure se è affetta.

Dato il peggioramento delle condizioni generali, si prendevano ripetuti contatti con vari specialisti del Centro di riferimento per chiedere un incontro per una rivalutazione clinica (ed anche per chiedere di effettuare la ERT presso il centro dialisi, nel tentativo di evitare al paziente ulteriori faticosi viaggi presso l'ambulatorio del centro di riferimento (oltre a quelli, necessari, al centro dialisi), senza riuscire tuttavia a trovare una data utile per l'incontro. Nel dicembre 2016, in previsione di intervento chirurgico di correzione della enterotomia, veniva sospeso il clopidogrel prescritto anni prima dal Centro di riferimento ma alcuni giorni dopo sopravveniva un episodio ischemico cerebrale transitorio con chiari segni seppur transitori di disorientamento spazio temporale e con evidente confusione mentale e compariva un dolore toracico retrosternale considerato aspecifico. Il paziente era ricoverato presso il Centro di riferimento per AF. La TC cerebrale evidenziava un sospetto emangioblastoma cerebellare inferiore sinistro ma anche aumento di dimensioni delle lesioni encefaliche già note. II paziente era dimesso dopo reintroduzione di clopidogrel e ranolazina con regressione della sintomatologia. Nei primi giorni di gennaio 2017 si ripresentava la sintomatologia neurologica con i medesimi segni clinici di disorientamento e confusione mentale e il paziente era inviato al DEA dove pochi giorni dopo decedeva per morte improvvisa da sospetta rottura di cuore (nessun riscontro diagnostico effet- 
tuato). La ERT era stata sospesa, per decisione improvvisa del Centro di riferimento di rimettere al Centro Dialisi la gestione della terapia ed al tempo stesso per le difficoltà tecniche in cui si era trovato quest'ultimo nel cercare di far acquistare in tempi rapidi il farmaco all'Azienda.

\section{Conclusione}

Questo caso clinico non solo esplicita l'andamento tempestoso che può manifestare l'AF in alcuni pazienti. Evidenzia anche come l'assenza (non infrequente nella pratica clinica quotidiana) di protocolli che prefigurino una collaborazione programmata fra singole figure specialistiche, ciascuna peraltro dotata di indubbia preparazione ed esperienza, possano limitare la gestione di pazienti con gravi e complesse patologie multisistemiche. In particolare, nel caso descritto, emergono le difficoltà che talora si incontrano nel concordare incontri collegiali per rivalutazioni cliniche come ad esempio sarebbe stato opportuno fra chirurghi, nefrologi, e medici del Centro di riferimento riguardo la possibilità di sospendere l'antiaggregante e sottoporre il paziente ad intervento di correzione della enterostomia, la mancanza di una decisione condivisa tra i vari specialisti su chi di essi dovesse farsi carico del paziente. Invece idealmente, un paziente così complesso avrebbe dovuto essere preso in carico da un team di specialisti messi in condizione di poter interagire tra loro in tempo quasi reale.

\section{Disclosures}

Financial support: No financial support was received for this submission.

Conflict of interest: The authors have no conflict of interest.

\section{Bibliografia}

1. Zizzo C, Monte I, Pisani A, et al. Molecular and clinical studies in five index cases with novel mutations in the GLA gene. Gene. 2016;578(1):100-4.

2. Colomba P, Nucera A, Zizzo $C$, et al. Identification of a novel mutation in the alpha-galactosidase $A$ gene in patients with Fabry disease. Clin Biochem. 2012;45(10-11):839-41. 